Gerontology
Received: January 7, 2015

Accepted: July 1, 2015

Published online: August 19, 2015

\title{
Cognitive Functions Buffer Age Differences in Technology Ownership
}

\author{
Stefan T. Kamin ${ }^{a} \quad$ Frieder R. Lang ${ }^{a, b}$ \\ ${ }^{a}$ Institute of Psychogerontology, Friedrich-Alexander University of Erlangen-Nuremberg, Nuremberg, and \\ ${ }^{b}$ German Institute for Economic Research, Berlin, Germany
}

\section{Key Words}

Technology use $\cdot$ Cognition · Interactions · Dominance analysis · Aging and technology · Gerontechnology

\begin{abstract}
Background: Technology plays a major role in enhancing quality of life and everyday competence in old age. Mechanic and pragmatic cognitive functions are known to serve as resources when using technology in everyday life. Not much is known about the differential role of mechanic and pragmatic cognitive functions when moderating reduced technology ownership in old age. Objective: In this research, we explored whether perceptual speed or verbal fluency is more important for buffering age differences in technology ownership. We investigate possible moderation effects of cognitive functions relative to demographic characteristics, socioeconomic status, and household composition variables. Methods: We report findings based on a nationally representative German sample of 3,357 younger and older adults between 18 and 94 years of age (mean $=51.2, \mathrm{SD}=$ 17.3). Interaction and relative importance analyses were conducted to examine the relative importance of perceptual speed and verbal fluency for the moderation of age differences in technology ownership across adulthood. Results: Findings suggest that perceptual speed $(B=0.0008, t=6.23$, $p<0.001)$ and verbal fluency $(B=0.0003, t=2.70, p<0.01)$ buffered age differences in technology ownership. The mod-
\end{abstract}

erating role of perceptual speed remained robust $(B=0.0007$, $t=5.48, p<0.001)$ when including interactions of age with demographic, socioeconomic, and household composition variables; however, the interaction between age and verbal fluency was no longer significant $(B=0.0002, t=1.82, p=$ 0.069). Relative importance analysis indicates that perceptual speed was the most important moderator of age differences ( $D W=0.0121$ ), whereas verbal fluency was less important for moderating the relation between age and technology ownership ( $D W=0.0039$ ). Conclusions: Mechanic and pragmatic cognitive functions may serve differently as moderators of the relation between age and technology ownership. Our findings suggest that perceptual speed was more important for buffering age differences in technology ownership than verbal fluency. Such findings underscore the relevance of information processing for the ownership of technological devices in late life.

(c) 2015 S. Karger AG, Basel

\section{Introduction}

Technology has been associated with quality of life in old age [1]. In general, two broad domains can be distinguished that show how older adults benefit from technology in everyday life [2]. The first pertains to the compensation or prevention of aging-related loss and decline, the second to the enrichment of life and enhancement of per-

\section{KARGER}

E-Mail karger@karger.com

www.karger.com/ger
(C) 2015 S. Karger AG, Basel

0304-324X/15/0622-0238\$39.50/0
Stefan T. Kamin

Institute of Pschogerontology, Friedrich-Alexander University Erlangen-Nuremberg Kobergerstrasse 62 DE-90408 Nuremberg (Germany)

E-Mail stefan.kamin@fau.de 
sonal competence. For example, there is evidence showing that the proactive use of hearing aids compensates negative effects of aging-related hearing loss [3]. On the other hand, innovative technology such as social media creates new opportunities for enhanced social contact and new meaningful experiences [4].

Opportunities of technological innovation are not without challenge for older adults. Learning to use new technology in old age often involves the investment of limited resources. Indeed, a variety of studies have shown that older adults are less likely to use new technology compared with younger persons $[5,6]$. In this context, findings have shown that cognitive functions play a major role in predicting technology use among older adults [7]. However, it is not well understood in what ways mechanic and pragmatic cognitive functions may serve differently as moderators of the relation between age and technology. Also, we do not yet understand which domains of cognitive ability are most important for explaining differences in technology use as people age [8]. Building on the two-component model of intellectual functioning [9], we explored the differential influence of perceptual speed (reflecting cognitive mechanics) and verbal fluency (reflecting cognitive pragmatics) on age differences in technology ownership in a nationally representative German sample of younger and older adults. Specifically, we investigated whether perceptual speed or verbal fluency is more important when moderating the relation between age and technology ownership. In addition to cognitive moderators, we also explored the relative importance of socioeconomic status, demographic characteristics, and household composition when explaining age differences in technology ownership.

Based on assumptions derived from the ecological model of aging [10], it is argued that technological environments are challenging for the older person of low competence $[11,12]$. Thus, having more personal resources available allows choosing from a greater number of technological resources in everyday life. In this research, we argue that cognitive functioning serves as a personal resource in technological environments. Research in this domain has shown that a multitude of specific cognitive abilities are involved when using technology in old age [7]. For example, findings from the CREATE study indicated a positive association between mechanic and pragmatic cognitive abilities and the use of everyday technology such as cellular phones or videocassette recorders [5]. In this study, it was also shown that cognitive abilities were associated with a broader experience of Internet and computer use. Similarly, other find-

Cognitive Functions Buffer Age

Differences ings that are based on representative data of older adults' ICT (information and communications technology) use suggested that mechanic cognitive functioning was associated with computer and Internet use [8]. Additional evidence relates to studies that have investigated task performance or problem solving when using specific technological devices and solutions $[13,14]$. In general, there is robust evidence that older adults with higher cognitive abilities may be better able to use technology in everyday life $[15,16]$. That is, transactions with technological demands in old age require the investment of behavioral and mental resources [17].

However, research in this domain has typically focused on explaining technology use from the perspective of different cognitive predictors in samples of older adults. Not much is known about the ways in which mechanic and pragmatic cognitive abilities may serve differently as moderators against reduced technology ownership in old age. When considering different trajectories of intellectual functioning across the life span, stronger declines of mechanic abilities are typically related to basic biomechanical processing operations, whereas stability or increases in pragmatic knowledge in everyday life may be associated with secular cultural changes over time $[18,19]$. One possible implication is that, in old age, the influence of mechanic abilities may become stronger with regard to the ownership of technology [5]. For example, knowledge-related verbal ability has been shown to predict the task performance on personal health records in a sample of middle-aged and older adults [14]. However, although older adults showed high levels of verbal ability they were less competent when using such devices. That is because the expression of pragmatic knowledge may depend on agerelated differences in mechanic abilities [9].

\section{The Present Study}

In this study, we hypothesize that perceptual speed plays a more important role for moderating age differences in technology ownership than verbal fluency. Clearly, ownership of technology is different from use of technology. In this study, we only have data on ownership, not actual everyday use of technology. In accordance with the ecological model of aging [10], we submit that technology ownership reflects the range of technological resources that are available to individuals, and that such resources are more likely to be used with stronger cognitive resources. Cognitive functioning is measured with short measures of perceptual speed and verbal fluency as marker abilities of mechanic and pragmatic cognitive functioning [20]. Perceptual speed is a key indicator of cogni- 
tive mechanics that is related to the capacity of information processing. There is considerable evidence that perceptual speed accounts for differences among other cognitive abilities [21]. Verbal fluency is known to indicate cognitive functions related to practical knowledge in everyday life [22].

Specifically, we explored the following hypotheses. First, we expected that both cognitive abilities would predict ownership of technological devices in the home environment. We also investigated the role of demographic and socioeconomic differences as well as the influence of household composition on technology ownership. In line with previous findings [5], we expected that older age and being female would be negatively associated with technology ownership. With regard to socioeconomic variables, findings have suggested that income and education were positively associated with technology use $[5,8]$. We expected to replicate such findings for technology ownership. Moreover, there is evidence that individuals have access to technology through other persons that may live in the same household [23]. Thus, we expected that technology ownership would be higher for people living together in the same household with a partner and/or children. Second, we expected buffering effects of cognitive abilities against lower technology ownership in old age. There is much evidence showing that members of different age cohorts have different knowledge of and experience with technological environments [24]. For instance, younger adults have often grown up with new technological developments, whereas older adults are often required to adapt to new technological demands. We assumed that adapting to such demands would require an increased investment of cognitive resources. Third, in line with theoretical assumptions of life span cognitive development, we hypothesized that perceptual speed would be more important for buffering age differences in technology ownership than verbal fluency. It is known that there is a strong interdependence of mechanic and pragmatic cognitive abilities across the life span $[9,25]$. For example, declines in mechanic functioning can be compensated with knowledge-related pragmatic ability. However, it is also known that age-related declines of mechanic functioning limit the expression of pragmatic knowledge. Thus, in late life, it becomes increasingly difficult to compensate for losses in mechanic functioning. Moreover, age-related slowing of information processing is often considered to be the primary mediator of age differences in cognitive functioning [21]. Finally, we explored the importance of cognitive moderators relative to demographic, socioeconomic, and household compo- sition variables that may also moderate the relation between age and technology ownership. We expected that the relative importance of perceptual speed would be robust above and beyond the effects of other moderators.

\section{Method}

Participants and Procedure

Analyses were performed on data from the German Socio-Economic Panel (SOEP) [26]. The SOEP is an ongoing nationally representative study of private households in Germany that began in 1984. We used data from a subsample in 2006 because this was the first time that indicators of cognitive functioning were assessed. The participants were at least 18 years old when interviewed in 2006 and provided all information on the variables in the study. SOEP data are hierarchical; that is, each person belongs to a household, which may result in dependent observations in the same cluster. In order to avoid such a dependency, we randomly selected one person from each household for the analyses. The final sample included 3,357 individuals between 18 and 94 years of age (mean $=51.2, \mathrm{SD}=17.3) .55 \%$ of the participants were female.

\section{Measures}

\section{Technology Ownership}

The SOEP includes questions about the ownership of different technological devices. Participants indicated whether a device was available in the home environment. We limited our analysis to technology that is related to the enrichment of daily life [2]. This included the ownership of five information and communication technology devices (cell phone, Internet, personal computer, DVD player, hi-fi stereo system). An overall score was generated by summing the number of available devices. The resulting score ranged from 0 to 5 devices (mean $=3.5, \mathrm{SD}=1.7$ ) and served as an indicator of technological resources available to the person. Cronbach's alpha was 0.80 for the scale.

Cognitive Functioning

The SOEP includes two ultrashort CAPI-based tests of cognitive functioning [27]. The Symbol Digit Test is a measure of perceptual speed. The participants in our study were required to match numbers with graphical symbols as quickly as possible. The number of correct responses after $90 \mathrm{~s}$ ranged from 1 to 60 (mean $=26.4, \mathrm{SD}=9.8)$ and reflected the capacity of processing speed. The Animal Naming task is a measure of verbal fluency. Participants in the study had to name as many different animals as possible during a time frame of $90 \mathrm{~s}$. The number of correct answers ranged from 1 to 86 ( mean $=25.3, \mathrm{SD}=11.1$ ) and served as an indicator of word fluency related to practical knowledge in everyday life.

\section{Socioeconomic Variables}

Socioeconomic variables included educational status and income. Educational status was specified as a dummy variable $(0=$ secondary school or below; 1 = university entrance diploma) and indicated that $25 \%$ of the participants had a university entrance diploma (German Fachabitur or Abitur). We included dummy variables of high-, middle-, and low-income groups. The variables 
Table 1. Correlations $(n=3,357)$

\begin{tabular}{|c|c|c|c|c|c|c|c|c|c|c|}
\hline (1) Age & - & & & & & & & & & \\
\hline (2) Female gender & 0.01 & - & & & & & & & & \\
\hline (3) Higher education & $-0.15^{* * *}$ & $-0.06^{* * *}$ & - & & & & & & & \\
\hline (4) Low income & $0.11^{* * *}$ & $0.08^{* * *}$ & $-0.20^{* * *}$ & - & & & & & & \\
\hline (5) Middle income & -0.01 & $-0.04^{*}$ & $-0.06^{* * *}$ & $-0.51^{* * *}$ & - & & & & & \\
\hline (8) Coresidence partner & 0.03 & $-0.09^{* * *}$ & 0.02 & $-0.41^{* * *}$ & $0.11^{* * *}$ & $0.31^{* * *}$ & $0.28^{* * *}$ & - & & \\
\hline (9) Perceptual speed & $-0.45^{* * *}$ & -0.00 & $0.19^{* * *}$ & $-0.14^{* * *}$ & -0.00 & $0.15^{* * *}$ & $0.13^{* * *}$ & 0.03 & - & \\
\hline (10) Verbal fluency & $-0.20^{* * *}$ & 0.01 & $0.21^{* * *}$ & $-0.15^{* * *}$ & $-0.04^{*}$ & $0.20^{* * *}$ & $0.09^{* * *}$ & $0.08^{* * *}$ & $0.44^{* * *}$ & - \\
\hline (11) Technology ownership & $-0.55^{* * *}$ & $-0.10^{* * *}$ & $0.29^{* * *}$ & $-0.44^{* * *}$ & $0.06^{* * *}$ & $0.39^{* * *}$ & $0.35^{* * *}$ & $0.24^{* * *}$ & $0.36^{* * *}$ & $0.26^{* * *}$ \\
\hline
\end{tabular}

were specified based on a tercile split of the monthly net household income. High income ranged from EUR 2,730 to 35,000 (mean = $4,412.9, \mathrm{SD}=2,469.2$ ), middle income ranged from EUR 1,604 to $2,700$ (mean $=2,148.3, \mathrm{SD}=310)$, and low income ranged from EUR 125 to $1,600($ mean $=1,153.4, \mathrm{SD}=330.2)$. People with low income were included as a reference group. Missing values were replaced with multiple imputations.

Household Composition

We included dummy variables indicating whether or not participants lived with a partner and/or with children (coresidence; $0=$ not living together; $1=$ living together). $61 \%$ of the participants reported living together with a partner; $30 \%$ of the participants reported living together with children.

\section{Data Analysis}

The current study is based on a cross-sectional approach. After reporting correlational data, we report findings from a series of hierarchical multiple regression models. We applied a stepwise approach and entered demographic, socioeconomic, and household composition variables in the first step, followed by cognitive predictors in the second step. In the third step, we entered interaction terms between age and cognitive predictors. Finally, we included interactions between age and demographic, socioeconomic, and household composition variables. All models were estimated using robust standard errors in order to account for heteroskedasticity and violations of distributional assumptions. The variables for the interaction terms were centered in order to make the models more interpretable. To better understand the differential influence of the variables in our study, we also computed general dominance weights for the main effects and residualized dominance weights for the interaction effects [28]. We used this approach because our variables were substantially correlated, thus calling into question the usefulness of standardized regression coefficients for addressing the relative importance of predictors. Moreover, addressing the overall contribution of a variable based on changes in explained variance is difficult because increments in variance are dependent on existing variables in a model. By contrast, a dominance weight reflects the unique impact of a variable and its impact in combination with other variables in the model [29]. As such, a dominance weight can be interpreted as a measure of relative variable importance. All reported analyses were conducted with STATA 13 [30]. Dominance weights were calculated with the DOMIN module [31].

\section{Results}

\section{Correlational Analysis}

Bivariate correlations were computed to explore the associations between the dependent and independent variables in the study. Table 1 shows that technology ownership was negatively correlated with increasing age, being female, and low income. We found positive correlations for higher education, middle and high income, residing with children or a partner in the same household, and cognitive functions. The correlation between cognitive functions and the number of reported devices was stronger for perceptual speed than for verbal fluency. It also shows different associations between the two cognitive domains and chronological age, suggesting age-related declines in perceptual speed and a greater stability of verbal fluency.

\section{Regression Models}

Table 2 shows the results of four regression models. We report unstandardized regression coefficients that indicate the change in technology ownership given a 1-unit increase in the respective predictors. First, we explored whether technology ownership could be predicted by demographic, socioeconomic, and household composition 
Table 2. Multiple regression models predicting technology ownership ( $\mathrm{n}=3,357)$

\begin{tabular}{|c|c|c|c|c|c|c|c|c|c|c|c|c|}
\hline & \multicolumn{3}{|l|}{ Model 1} & \multicolumn{3}{|l|}{ Model 2} & \multicolumn{3}{|l|}{ Model 3} & \multicolumn{3}{|l|}{ Model 4} \\
\hline \multicolumn{13}{|l|}{ Sociodemographic variables } \\
\hline Female & $-0.1997^{* * *}$ & 0.04 & -0.06 & $-0.2085^{* * *}$ & 0.04 & -0.06 & $-0.2009^{* * *}$ & 0.04 & -0.06 & $-0.1518^{* * *}$ & 0.04 & -0.05 \\
\hline \multicolumn{13}{|l|}{ Socioeconomic variables } \\
\hline Higher education & $0.4940^{* * *}$ & 0.05 & 0.13 & $0.4394^{* * *}$ & 0.05 & 0.11 & $0.4421^{* * *}$ & 0.05 & 0.11 & $0.4411^{* * *}$ & 0.05 & 0.11 \\
\hline High income & $1.3125^{* * *}$ & 0.06 & 0.37 & $1.2644^{* * *}$ & 0.06 & 0.35 & $1.2273^{* * *}$ & 0.06 & 0.34 & $1.1910^{* * *}$ & 0.06 & 0.33 \\
\hline \multicolumn{13}{|l|}{ Household composition } \\
\hline Coresidence children & $0.3925^{* * *}$ & 0.05 & 0.11 & $0.3990^{* * *}$ & 0.05 & 0.11 & $0.3677 * * *$ & 0.05 & 0.10 & $0.5333^{* * *}$ & 0.06 & 0.15 \\
\hline Coresidence partner & $0.2776^{* * *}$ & 0.05 & 0.08 & $0.2675^{* * *}$ & 0.05 & 0.08 & $0.2410^{* * *}$ & 0.05 & 0.07 & $0.2863^{* * *}$ & 0.05 & 0.08 \\
\hline \multicolumn{13}{|l|}{ Cognitive functions } \\
\hline Perceptual speed & & & & $0.0089^{* * *}$ & 0.00 & 0.05 & $0.0108^{* * *}$ & 0.00 & 0.06 & $0.0106^{* * *}$ & 0.00 & 0.06 \\
\hline Age $\times$ high income & & & & & & & & & & $0.0167^{* * *}$ & 0.00 & 0.08 \\
\hline Age $\times$ education & & & & & & & & & & $0.0091^{* *}$ & 0.00 & 0.04 \\
\hline Age $\times$ coresidence child & & & & & & & & & & $0.0283^{* * *}$ & 0.00 & 0.12 \\
\hline Age $\times$ female & & & & & & & & & & $-0.0064^{* *}$ & 0.00 & -0.05 \\
\hline Intercept & $2.4858^{* * *}$ & 0.05 & & $2.5284^{* * *}$ & 0.05 & & $2.6439^{* * *}$ & 0.05 & & $2.6384^{* * *}$ & 0.05 & \\
\hline Adjusted $\mathrm{R}^{2}$ & & & 0.50 & & & 0.51 & & & 0.52 & & & 0.54 \\
\hline
\end{tabular}

$\mathrm{B}=$ Unstandardized coefficient; $\mathrm{SE}=$ robust standard error; $\beta=$ standardized beta coefficient. ${ }^{*} \mathrm{p}<0.05 ;{ }^{* *} \mathrm{p}<0.01 ;{ }^{* * *} \mathrm{p}<0.001$.

variables (model 1). The number of reported devices significantly decreased for each year of age and being female and significantly increased for higher education, middle and high income, and residing with children or a partner in the same household. We extended the model by including cognitive functions and found significant main effects of perceptual speed and verbal fluency, indicating increased ownership with each correct response on the performance measures (model 2). Next, we tested our hypothesis on possible buffering effects of cognitive functions (model 3). This model provided significant age interactions with regard to perceptual speed and verbal fluency. These interactions indicated that higher levels of both cognitive domains were associated with a reduction in age differences in technology ownership. Finally, we investigated whether cognitive moderators would be robust when taking into account demographic, socioeconomic, and household composition variables that may also moderate the association between age and technology ownership (model 4). For the purpose of clarity, we show only the final model with the significant age interactions. Findings suggest that higher technology ownership in old age was associated with high income, high education, and residing with chil- dren in the same household. The negative association between age and technology ownership was stronger for women. Perceptual speed remained stable as a moderator of age differences; however, the interaction between age and verbal fluency was no longer significant.

It should be noted that Internet and computer ownership were strongly related. Although there was a significant proportion of people who reported computer but not Internet ownership, there were only few people who reported Internet but not computer ownership. We collapsed Internet and computer ownership and repeated all analysis with only five levels of technology ownership in order to ensure that this dependency was not biasing the estimates. Our findings remained robust.

\section{Relative Importance Analysis}

Table 3 provides the dominance weights for each variable and interaction based on the final regression model. The ranking reflects the relative importance of variables based on their dominance weights. Unlike in regression, interaction terms can be directly compared with their associated main effects. We also present the incremental change in explained variance for comparison purposes. 
Table 3. Relative importance analysis $(n=3,357)$

\begin{tabular}{lrrr}
\hline & DW & Rank & $\Delta \mathrm{R}^{2}$ \\
\hline Main effects & & & \\
$\quad$ Age & 0.1994 & 1 & 0.2987 \\
Female & 0.0050 & 13 & 0.0079 \\
Higher education & 0.0360 & 5 & 0.0425 \\
Middle income & 0.0214 & 7 & 0.0042 \\
High income & 0.0909 & 2 & 0.1319 \\
Coresidence child & 0.0483 & 3 & 0.0140 \\
Coresidence with partner & 0.0262 & 6 & 0.0048 \\
Perceptual speed & 0.0465 & 4 & 0.0042 \\
$\quad$ Verbal fluency & 0.0206 & 8 & 0.0024 \\
Interaction effects & & & \\
Age $\times$ speed & 0.0121 & 9 & 0.0090 \\
Age $\times$ fluency & 0.0039 & 14 & 0.0009 \\
Age $\times$ high income & 0.0080 & 11 & 0.0070 \\
Age $\times$ education & 0.0068 & 12 & 0.0008 \\
Age $\times$ coresidence child & 0.0105 & 10 & 0.0073 \\
Age $\times$ female & 0.0029 & 15 & 0.0009 \\
\hline
\end{tabular}

DW $=$ Dominance weights for main effects and residualized dominance weights for interaction effects that may only be compared with caution; rank $=$ rank ordering of variable importance based on dominance weights; $\Delta \mathrm{R}^{2}=$ incremental change in $\mathrm{R}^{2}$.

When comparing main effects only, it can be seen that age, high income, coresidence with children, and perceptual speed were the most important predictors of technology ownership, followed by higher education and coresidence with partner. Although the standardized regression coefficients suggested small differences between both cognitive predictors, the dominance weights indicated that verbal fluency was less important than perceptual speed in predicting technology ownership. Gender was the least important predictor in the model. With regard to the interaction effects, we found that perceptual speed was the most important moderator of age differences, followed by coresidence with children, high income, and higher education. Verbal fluency and being female were less important as moderators of age differences.

\section{Discussion}

We explored associations between cognitive functions, demographic characteristics, socioeconomic variables, household composition, and technology ownership in a nationally representative German sample of younger and older adults. Findings suggest that interin- dividual differences in cognitive speed and verbal fluency differently predicted the number of diverse technological devices owned across adulthood. Notably, the relative importance of perceptual speed was around twice as large as verbal fluency when predicting technology ownership. Moreover, findings show that perceptual speed and verbal fluency served differently as moderators of the relation between age and technology. The interaction patterns suggest that both cognitive abilities buffered age differences in technology ownership. However, findings point to a primacy of perceptual speed, indicating that information processing was more important for buffering age differences than verbal fluency. We further compared the moderating role of cognitive functions with age interactions of demographic, socioeconomic, and household composition variables. Again, perceptual speed was the strongest moderator of age differences, whereas the interaction between age and verbal fluency was no longer significant.

Our findings further confirm that individual differences in cognitive functioning are associated with the ownership of technology in old age $[5,8]$. Research in this domain has contributed to an improved understanding of the role of a broad multitude of cognitive constructs; for example, with regard to task switching [16] or executive functions [15]. From the perspective of cognitive aging, we argue that declines in such abilities may reflect an age-related slowing of information processing [21]. Accordingly, our research shows the relevance of information processing with regard to technology ownership in old age as compared with verbal fluency. This finding relates to assumptions of life span cognitive development research that suggests that in old age, the role of mechanic or fluid aspects of cognition becomes stronger [25]; for example, with regard to everyday competence [32] or learning ability [33]. To be clear, we contend that an improved understanding of the relation between age and technology requires the consideration of different mental and behavioral resources including practical and experience-related knowledge. However, our findings suggest that processing speed may be a key indicator for better understanding different patterns of technology ownership across adulthood.

Such results may have implications for the successful development of innovative technology in old age. For designers and engineers, it is crucial to know who the users are and how to involve them in the development process [34]. This requires the efficient and precise measurement of cognitive, behavioral, and physical abilities for involving relevant persons in different phases of the develop- 
ment process. In this context, it is often not possible to conduct extensive assessments of different psychometric and cognitive variables. Thus, knowing what abilities of cognitive functioning are most important for explaining competent technology use in old age may be helpful for conducting appropriate and economic assessments. Moreover, we submit that there is a need to improve understanding of the interplay between different cognitive functions in technology development for older adults. In this study, perceptual speed and verbal fluency were both predictors of technology ownership; and even though older adults had stronger pragmatic than mechanic cognitive resources, the influence of pragmatic functioning was negligible with regard to technology ownership in old age. Practically speaking, an engineer may correctly assume that older adults may benefit from verbal cues because of the relative stability of pragmatic resources; however, processing verbal information in old age may also depend on the capacity of information processing, which should be considered in product development.

Although we have focused on cognitive functions, findings may also shed light on the role of socioeconomic factors associated with technology ownership. Specifically, we found that income and education were major predictors of technology ownership, replicating previous findings in the literature $[5,8]$. In addition, we observed buffering effects of both variables, suggesting that older adults with higher income and education owned more technological devices as compared with those who had less socioeconomic resources available. Such findings may reflect cohort-specific effects because older adults in this sample were on average less educated and had lower income than members of younger age cohorts. With regard to household composition, we found that technology ownership was higher for older adults who reported living together with children. Previous findings have found evidence for an association between social integration and technology use in old age [8]. Accordingly, we submit that social relationships may serve as compensatory resources in technological environments (e.g. through social support), particularly in the face of agingrelated physical and cognitive losses. Although this finding should be interpreted with caution given the limited usefulness of technology ownership as a measure of actual use, it may be suggested that the technological environment is richer for older persons who live together with children.

Finally, we found gender differences, indicating that in late life technology ownership was lower among women. Again, we suggest that this finding reflects cohort-specif- ic effects that may be associated with historical role models in Germany [35]. The central role of men in older age cohorts was more strongly focused on the occupational world, often requiring technological knowledge. By contrast, role expectations for women were associated with being a housewife and mother. One implication is that gendered roles in older adults were associated with different experiences with technological environments, which may have influenced actual technology ownership. In this context, access to technology among older women may also depend more strongly on marital or partnership status. Future research may improve our understanding of how gender roles and socialization experiences are associated with technology ownership. However, it should be noted that main and interaction effects involving gender were relatively small and less important compared with other variables in the study.

This study has limitations that ought to be considered when interpreting our findings. The regression models indicated that age was a major predictor of technology ownership, even when accounting for cognitive, demographic, socioeconomic, and household variables. In accordance with the literature [11], we argue that there is a broad range of factors associated with age differences in technology ownership. For example, motivational resources related to subjective technology adaptivity have been found to predict technology use among older adults [36]. A promising route of future research may be to explore the interdependence between cognitive and motivational resources in technological environments in old age. For example, motivational resources may serve to compensate for limited cognitive ability when using new technology.

The ownership of technological devices in the home environment is a limited measure of technology use. Nothing can be said about the actual frequency of use, which may raise questions about the ecological validity of this measure. Also, we are aware that ownership of technology implies that all members of a household may be users, and that controlling for children or partner in the same household may not rule out the possibility that devices may not be used by the participants in our study. Moreover, our measure of technology ownership included only five devices, which may raise the question of whether this outcome should be treated as a continuous variable. Clearly, there is a need to test our hypothesis on the primacy of perceptual speed with experimental approaches, including innovative measures such as the day reconstruction method, which has been recently used in the context of technology and aging [3]. Such studies may 
also focus more specifically on the interplay between the person and the technological environment. Based on the ecological model, one could also expect that with very high competence, technological resources may become irrelevant when there is no challenge. For example, older adults with strong cognitive resources may be more likely to value mobile Internet access, thus rejecting cell phones without this functionality. Although we analyzed devices in this study that were relatively new in 2006, it was not possible for us to determine the actual extent of technological demands associated with the breadth of ownership at this time. Future studies may systematically vary technological demands, for example, by comparing cutting-edge innovations with more traditional ICT solutions in order to better understand the influence of cognitive functions relative to technological demands among younger and older adults.

Our research included only two measures of cognitive ability. In accordance with the literature [20], we argue that perceptual speed and verbal fluency reflect generally accepted ability markers of cognitive functioning. However, we submit that further exploring the role of perceptual speed as compared with other cognitive abilities may require the integration of additional measures related to reasoning, working memory, and practical knowledge [37]. Such research may also apply other theories of intellectual functioning such as the Cattell-Horn-Carroll approach that proposes a broader range of different cognitive abilities [38]. The ultra-short tests in the SOEP were developed based on the two-component model, and that is why we have used this approach in this article. Also, the two-component model is commonly used in aging research [9].

Measures of cognitive functioning may have limitations in the context of survey research. Although the ultra-short tests in the SOEP have been found to provide valid and reliable assessments of cognitive performance [27], it is possible that ecological validity in the home environment may be an issue. For example, we do not know if interruptions (e.g. doorbell, phone call) may have influenced the test situation. Also, we do not know whether or not participants had visual impairments or motor difficulties that may have affected the test scores. Moreover, some of our participants had very low scores on the cognitive measures, which may be an indicator of cognitive impairments such as dementia. We did not exclude persons with low scores on the measures because of two reasons. First, we had no information about a clinical diagnosis of cognitive impairment. Ultra-short tests of cognitive ability as applied in the SOEP do not have the diagnostic accuracy that is needed for the assessment of cognitive impairments. Second, excluding participants that may potentially suffer from cognitive impairments may have questioned the generalizability of age differences in this nationally representative dataset.

Finally, we included cross-sectional data only. Therefore, not much can be said about the extent to which the findings reflect an interaction between general developmental changes and different formative experiences. In addition, cross-sectional designs do not account for within-person change when considering differential effects of cognitive abilities with regard to technology ownership across the life span. A better understanding of age differences in technology ownership would require the implementation of longitudinal studies.

Our findings are in accordance with the idea that chronological age alone has little impact on the relation between the person and his or her technological environment [39]. Instead, it might be the interaction between socialization experiences in technological environments, the availability of socioeconomic resources, and developmental change that affects technology ownership in old age. One implication is that at any given period of time, individuals have different experiences with particular technological environments. As individuals grow older, technological developments may change their environments. Such changes might not be compatible with the experiences and needs of older age cohorts [40]. At the same time, cognitive aging and socioeconomic discrepancies may place additional constraints on the ownership of new technological resources. Indeed, findings indicated a reduction of age differences in technology ownership when accounting for the buffering effects of cognitive and socioeconomic variables. Taken together, our findings suggest that perceptual speed was a more important moderator of age differences in technology ownership than verbal fluency or socioeconomic variables. We hope that our findings may contribute to an improved understanding of the ownership of technology in old age.

\section{Acknowledgements}

This research was supported by the German Federal Ministry of Education and Research (16SV5703). We thank Bettina Williger, Jane Zagorski, and Jeremy Hamm for valuable comments on the manuscript.

\section{Disclosure Statement}

The authors state that there are no conflicts of interests. 


\section{References}

1 Schulz R, Wahl H, Matthews JT, De Vito Dabbs A, Beach SR, Czaja SJ: Advancing the aging and technology agenda in gerontology. Gerontologist DOI: 10.1093/geront/gnu071.

$>2$ Bouma H: Foundations and goals of gerontechnology. Gerontechnology 2012;11:1-4.

-3 Williger B, Lang FR: Hearing aid use in everyday life: managing contextual variability. Gerontology 2015;61:158-165.

$\checkmark 4$ Leist AK: Social media use of older adults: a mini-review. Gerontology 2013;59:378-384.

$\checkmark 5$ Czaja SJ, Charness N, Fisk AD, Hertzog C, Nair SN, Rogers WA, et al: Factors predicting the use of technology: findings from the Center for Research and Education on Aging and Technology Enhancement (CREATE). Psychol Aging 2006;21:333-352.

6 Gell NM, Rosenberg DE, Demiris G, LaCroix AZ, Patel KV: Patterns of technology use among older adults with and without disabilities. Gerontologist 2015;55:412-421.

7 van der Wardt V, Bandelow S, Hogervorst E: The relationship between cognitive abilities, well-being and use of new technologies in older people. Gerontechnology 2013;10:187207.

$>8$ Elliot AJ, Mooney CJ, Douthit KZ, Lynch MF: Predictors of older adults' technology use and its relationship to depressive symptoms and well-being. J Gerontol B Psychol Sci Soc Sci 2014;69:667-677.

$\checkmark 9$ Baltes PB, Lindenberger U, Staudinger UM: Life span theory in developmental psychology; in Damon W, Lerner RM (eds): Handbook of Child Psychology. Vol 1. Theoretical Models of Human Development, ed 6. New York, Wiley, 2006, pp 569-664.

10 Lawton MP: Behavior relevant ecological factors; in Schaie KW, Schooder C (eds): Social Structure and Aging. Hillsdale, Erlbaum, 1989, pp 57-77.

11 Rogers WA, Fisk AD: Toward a psychological science of advanced technology design for older adults. J Gerontol B Psychol Sci Soc Sci 2010;65:645-653.

12 Wahl H, Mollenkopf $\mathrm{H}$ : Impact of everyday technology in the home environment of older adults quality of life; in Charness N, Schaie KW (eds): Impact of Technology on Successful Aging. New York, Springer, 2003, pp 215 241.

-13 Czaja SJ, Sharit J, Lee CC, Nair SN, Hernández MA, Arana N, et al: Factors influencing use of an e-health website in a community sample of older adults. J Am Med Inform Assoc 2013;20:277-284.
14 Taha J, Czaja SJ, Sharit J, Morrow DG: Factors affecting usage of a personal health record (PHR) to manage health. Psychol Aging 2013; 28:1124-1139.

15 Fazeli PL, Ross LA, Vance DE, Ball K: The relationship between computer experience and computerized cognitive test performance among older adults. J Gerontol B Psychol Sci Soc Sci 2013;68:337-346.

16 Tun PA, Lachman ME: The association between computer use and cognition across adulthood: use it so you won't lose it? Psychol Aging 2010;25:560-568.

17 Lindenberger U, Lövdén M, Schellenbach M, Li S, Krüger A: Psychological principles of successful aging technologies: a mini-review. Gerontology 2008;54:59-68.

18 Lindenberger U, Reischies FM: Limits and potentials of intellectual functioning in old age; in Baltes PB, Mayer KU (eds): The Berlin Aging Study: Aging from 70 to 100. Cambridge, Cambridge University Press, 1999, pp 329-359.

19 Schaie KW: Intellectual Development in Adulthood: The Seattle Longitudinal Study. New York, Cambridge University Press, 1996.

20 Lindenberger U, Baltes PB: Intellectual functioning in old and very old age: cross-sectional results from the Berlin Aging Study. Psychol Aging 1997;12:410-432.

21 Salthouse TA: The processing-speed theory of adult age differences in cognition. Psychol Rev 1996;103:403-428.

22 Lindenberger U, Mayr U, Kliegl R: Speed and intelligence in old age. Psychol Aging 1993;8: 207-220.

-23 Selwyn N, Gorard S, Furlong J, Madden L: Older adults' use of information and communications technology in everyday life. Ageing Soc 2003;23:561-582.

24 Fozard JL, Wahl H: Age and cohort effects in gerontechnology: a reconsideration. Gerontechnology 2012;11:10-21.

25 Baltes PB, Staudinger UM, Lindenberger U: Lifespan psychology: theory and application to intellectual functioning. Annu Rev Psychol 1999;50:471-507.

26 Wagner GG, Frick JR, Schupp J: The German Socio-Economic Panel Study (SOEP) - scope, evolution and enhancements. Schmollers Jahrb 2007;127:139-169.

27 Lang FR, Weiss D, Stocker A, von Rosenbladt $\mathrm{B}$ : Assessing cognitive capacities in computer-assisted survey research: two ultra-short tests of intellectual ability in the German Socio-Ecomomic Panel (SOEP). Schmollers Jahrb 2007;127:183-192.
28 LeBreton JM, Tonidandel S, Krasikova DV: Residualized relative importance analysis: a technique for the comprehensive decomposition of variance in higher order regression models. Organ Res Methods 2013;16:449473.

29 Tonidandel S, LeBreton JM: Relative importance analysis: a useful supplement to regression analysis. J Bus Psychol 2011;26:1-9.

30 StataCorp: STATA Statistical Software, release 13. StataCorp LP, 2013.

31 Luchman JN: DOMIN: Stata module to conduct dominance analysis. 2013.

32 Baltes MM, Wilms H, Borchelt M: Everyday competence in old age and very old age: theoretical considerations and empirical findings; in Baltes PB, Mayer KU (eds): The Berlin Aging Study: Aging from 70 to 100. Cambridge, Cambridge University Press, 1999, pp 384-402.

33 Baltes PB, Kliegl R: Further testing of limits of cognitive plasticity: negative age differences in a mnemonic skill are robust. Dev Psychol 1992;28:121-125.

34 Fisk AD, Rogers WA, Charness N, Czaja SJ, Sharit J: Designing for Older Adults: Principles and Creative Human Factors Approaches, ed 2. Human Factors and Aging Series. Boca Raton, CRC Press, 2009.

-35 Geissel B: Germany: successful quota rules in a gendered society; in Dahlerup D, Leijenaar M (eds): Breaking Male Dominance in Old Democracies. Oxford, Oxford University Press, 2013, pp 197-218.

36 Kamin ST, Lang FR: The Subjective Technology Adaptivity Inventory (STAI): a motivational measure of technology usage in old age. Gerontechnology 2013;12:16-25.

37 Salthouse T: Consequences of age-related cognitive declines. Annu Rev Psychol 2012; 63:201-226.

38 McGrew KS: The Cattell-Horn-Carroll theory of cognitive abilities: past, present, and future; in Flanagan DP, Harrison PL (eds): Contemporary Intellectual Assessment: Theories, Tests, and Issues. New York, Guilford Press, 2005, pp 136-181.

39 Broady T, Chan A, Caputi P: Comparison of older and younger adults' attitudes towards and abilities with computers: implications for training and learning. Br J Educ Technol 2010;41:473-485.

40 Lawton MP: Future society and technology; in Graafmans J, Taipale V, Charness N (eds): Gerontechnology: A Sustainable Investment in the Future. Amsterdam, IOS Press, 1998, pp 12-22. 